tens of millions of such pairs are thought to have formed in each galaxy over the age of the Universe $^{3}$. However, most of these systems either break up as the black holes form, or are created so widely apart that they do not have enough time to merge.

There are three main theories for how black holes can come together into a bound pair that merges within the age of the Universe. One scenario is that the black holes form close together, resulting from the collapse of massive stars that have undergone coupled stellar evolution ${ }^{4}$. The spins of these black holes would tend to be relatively high ${ }^{5}$ and would have a propensity to be aligned. If such spins were observed, we could infer that there is a mechanism for black-hole pairs to form in close proximity. This would tell us how massive stars evolve and die, in isolation, to become black holes.

Another possibility is that the black holes form separately, in crowded stellar environments ${ }^{6}$. Such black holes are then brought closer together because of gravitational (dynamical) interactions with other astronomical objects, before merging. In this case, the spins of the black holes could either be low or high, and most would be misaligned.

A third option is that the black holes were produced in the early Universe $\mathrm{C}^{7}$. This scenario assumes that in the extremely hot, dense plasma that existed shortly after the Big Bang, there were large fluctuations in the density of particles making up the Universe, due to physics beyond that of the standard model of particle physics. Particularly dense regions would directly collapse into black holes that would subsequently merge. The spins of such primordial black holes, if they exist, are likely to be relatively low and misaligned.

Farr and colleagues examined the four probable detections of gravitational waves that have so far been reported by Advanced LIGO $^{1,8-10}$, and analysed the magnitude and alignment of the spins of the black holes responsible for the signals. Despite the limited data, the authors found that the signals are already suggestive of black holes whose spins are relatively high and randomly aligned. Their results therefore favour the theory that systems of orbiting black holes form by dynamical evolution.

However, more merger detections are needed to confirm Farr and colleagues' findings, because there are uncertainties in the spin measurements for each black-hole pair. The examples we have observed so far could be atypical (due to random chance), and there might be multiple formation mechanisms for these systems. Nevertheless, the authors demonstrate that if one mechanism is dominant, as few as ten additional detections should be sufficient to start ruling out other formation theories.

With improved sensitivity and extended observations, Advanced LIGO should discover more merging black holes in the next few years. As the statistics of the detected population build up, the competing formation theories will be tested and some models will be ruled out. More data will be needed to test more-complicated scenarios, such as those involving three black holes or black holes that receive kicks from supernova explosions during their formation that result in spin-orbit misalignment. Nevertheless, Farr and colleagues' results are important because they tell us how many data are needed to test the main formation theories, and show that the number of required observations is likely to be achieved in the near future.

Steinn Sigurðsson is in the Department of Astronomy and Astrophysics, Pennsylvania
State University, Pennsylvania 16802, USA. e-mail:steinn@astro.psu.edu

1. Abbott, B. P. et al. Phys. Rev. Lett. 116, 061102 (2016).

2. Farr, W. M. et al. Nature 548, 426-429 (2017).

3. Elbert, O. D., Bullock, J. S. \& Kaplinghat, M. Preprint at https://arxiv.org/abs/1703.02551 (2017).

4. Belczynski, K., Holz, D. E., Bulik, T. \& O'Shaughnessy, R. Nature 534, 512-515 (2016).

5. Miller, M. C. \& Miller, J. M. Phys. Rep. 548, 1-34 (2015).

6. Rodriguez, C. L., Haster, C.-J., Chatterjee, S., Kalogera, V. \& Rasio, F. A. Astrophys. J. Lett. 824, L8 (2016).

7. Bird, S. et al. Phys. Rev. Lett. 116, 201301 (2016).

8. Abbott, B. P. et al. Phys. Rev. Lett. 116, 241103 (2016).

9. Abbott, B. P. et al. Phys. Rev. X 6, 041015 (2016).

10.Abbott, B. P. et al. Phys. Rev. Lett. 118, 221101 (2017).

\title{
BIOTECHNOLOGY
}

\section{At the heart of gene edits in human embryos}

The gene-editing technology CRISPR-Cas has been used in human embryos grown in vitro to correct a disease-associated mutation. The introduction of editing components at fertilization aided repair efficiency. SEE ARTICLE P.413

\section{NERGES WINBLAD \& FREDRIK LANNER}

I The ability to selectively edit targeted genome regions using a technique known as CRISPR-Cas editing has transformed many areas of biological research. These advances have raised the question of whether this technique will be used in the clinic in future to treat or prevent disease. Clinical trials using this technique to edit human cells are already under way, for example testing the use of edited immune cells to treat cancer ${ }^{1}$. There is ongoing debate about the potential use of CRISPR-Cas to modify the human genome in an individual, a subject that raises many ethical and regulatory issues ${ }^{2}$. And if this were to occur, what extra scientific research would be necessary to reach the stage at which this approach could be implemented in the clinic? On page 413, Ma et al. ${ }^{3}$ report the use of CRISPR-Cas editing to repair a gene alteration associated with heart disease, in a study of human embryos grown in vitro. The authors thoroughly analyse the edited embryos, demonstrating that some technical hurdles often associated with using such genomeediting technology might be preventable.

The condition known as hypertrophic cardiomyopathy is an inherited cardiac disease. It can be caused by mutations in many different genes, including cardiac myosin-binding protein $\mathrm{C}$ (MYBPC3). This gene encodes a protein that contributes to the structural maintenance of heart muscle and the regulation of its contraction and relaxation ${ }^{4}$. The presence of one mutant copy of MYBPC3 causes symptoms that usually manifest as heart failure. Although existing treatments can lessen the symptoms, there is no way of tackling the underlying genetic cause in a patient ${ }^{5}$. Ma and colleagues investigated the use of gene editing to correct this mutation.

One option to prevent some mutationassociated conditions from being inherited is to use genetic testing during in vitro fertilization (IVF) fertility treatment. This enables the selection of embryos for implantation that do not contain the specific mutation. If one of the parents participating in IVF treatment had a mutant copy of MYBPC3, $50 \%$ of the couple's fertilized embryos would inherit the condition. Ma and colleagues propose a hypothetical scenario in which their approach could enable an individual with hypertrophic cardiomyopathy to increase the percentage of embryos available for IVF implantation that would not inherit the disease.

In the past few years, CRISPR-Cas has been developed ${ }^{6,7}$ to efficiently and precisely edit the human genome. Although this method has been widely adopted using mammalian cellculture systems and animal embryo models, only three published studies ${ }^{8-10}$ report the use of this technique in human embryos.

The CRISPR-Cas editing system needs just two components to modify DNA: a guide 
RNA sequence and a Cas nuclease enzyme, with Cas9 the most commonly used. The specific genomic target is determined by the guide RNA, which forms a complex with Cas9, enabling the enzyme to target a genomic site that contains a matching sequence. This is where Cas9 cleaves the DNA, causing a double-stranded break ${ }^{6,7}$.

The formation of double-stranded breaks can activate one of the two main DNA-repair pathways in the cell: either the non-homologous end joining (NHEJ) pathway or the homology-directed repair (HDR) pathway. The NHEJ pathway repairs a break by randomly adding or deleting nucleotides, which results in changes to the DNA sequence, making it unsuitable for gene-correction purposes. Therefore, approaches focus on HDR, which uses homologous (matching) sequences to repair the DNA breaks, making it possible to introduce specific sequences to enable tailored repair.

Timed delivery of CRISPR components at an appropriate point in the cell's division cycle - either at the transition between the G1 and $S$ phases or at the transition between $\mathrm{G} 2$ and $\mathrm{M}$ phases - might lead to preferential use of the HDR pathway ${ }^{11}$. Unfortunately, in previous reports, the efficiency of HDR repair after CRISPR-Cas9 action has been undesirably low both in cultured human embryonic stem cells (around 2\% efficiency) ${ }^{11}$ and in the human embryo (14.3-25\% efficiency) ${ }^{8}$.

$\mathrm{Ma}$ and colleagues created CRISPR-Cas9 gene-editing constructs to target $M Y B P C 3$, and verified and analysed the gene targeting using human stem cells. They then began to work with human embryos. One of the main challenges of using CRISPR-Cas9 to edit humanembryo genomes has been the phenomenon of mosaicism, in which gene-editing inefficiencies result in a developing embryo that has both edited and unedited cells ${ }^{8-10}$ (Fig. 1a). This might lead to a mixture of healthy and diseased cells in various tissues and organs, possibly causing disease symptoms.

Ma et al. investigated a situation in which the father had one mutant copy of MYBPC3 and the mother had only wild-type copies of the gene. In control experiments, $47.4 \%$ ( 9 out of 19) of embryos fertilized in vitro did not inherit a mutant copy of $M Y B P C 3$, the proportion expected given that half of the sperm should have the wild-type copy of the gene. The authors demonstrated that, if genomeediting components were injected together with sperm into a human egg (oocyte) at a stage in the oocyte cell cycle known as metaphase II (Fig. 1b), then $72.4 \%$ of the resulting embryos (42 out of 58) had only the wild-type version of $M Y B P C 3$. The other 16 embryos had copies of $M Y B P C 3$ with signs of NHEJ-mediated editing that targeted the mutant version of the gene but did not repair it to the wild-type version.

Previous studies of CRISPR-Cas editing in human embryos ${ }^{8-10}$ added the gene-editing
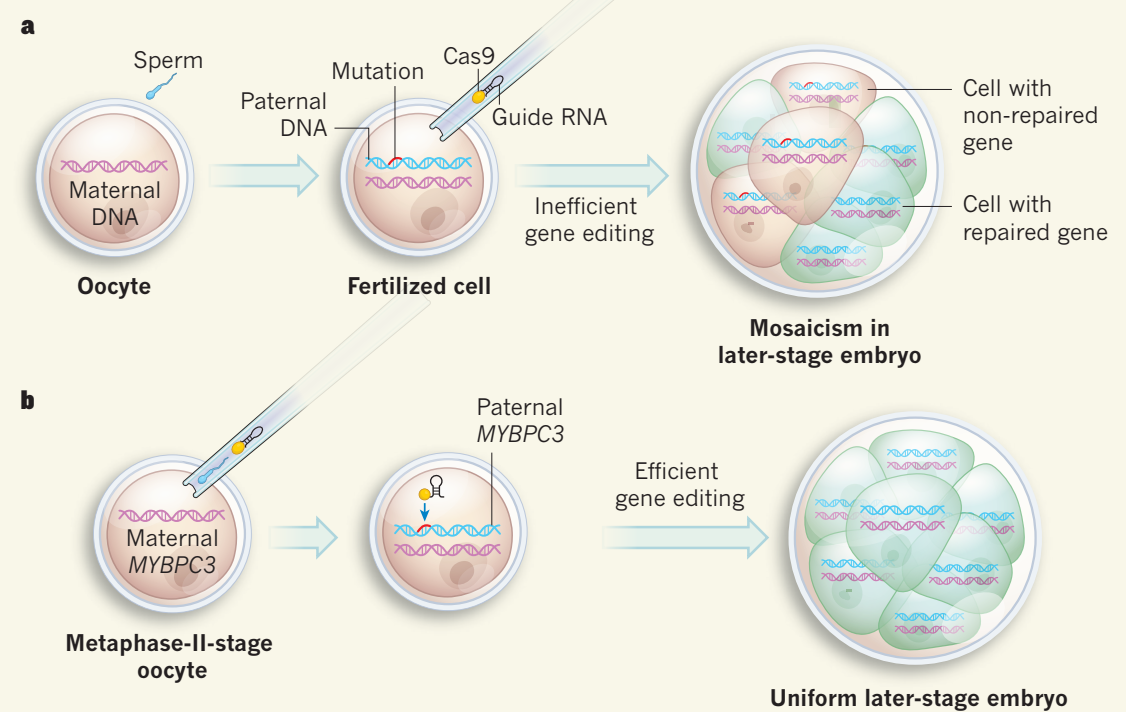

Figure 1 | Gene editing in embryos. a, The gene-editing system ${ }^{6,7}$ CRISPR-Cas can repair a mutation in a gene. In the three previous published studies ${ }^{8-10}$ using this technique in human embryos grown in vitro, an egg (oocyte) was fertilized by sperm and then the editing components were injected into the cell. These include the enzyme Cas 9 and a guide RNA sequence that helps to direct the editing machinery to a specific location in the genome, for example to repair a mutation inherited from the father. However, editing is often inefficient, and later-stage embryos can contain a mixture of repaired and non-repaired cells - a phenomenon known as mosaicism. b, Ma et al. ${ }^{3}$ took an alternative approach to correct a mutation in the MYBPC3 gene (which is associated with heart disease) in human embryos grown in vitro. They injected gene-editing components and sperm into oocytes that contained non-mutated versions of $M Y B P C 3$. Half the sperm used had a MYBPC 3 mutation. The oocytes were injected at the metaphase II stage of their cell cycle. Ma et al. report that 42 of 58 embryos tested (72.4\%) did not have the MYBPC3 mutation, and their analysis suggests that the maternal copy of the gene is used as a template to guide the repair. This approach resulted in efficient, uniform gene editing in embryos, which progressed to reach a later stage of embryonic development.

components after fertilization. The low levels of mosaicism (one of the 42 embryos with wild-type $M Y B P C 3$ was a mosaic of cells that each had one of two versions of the wildtype gene) described by Ma et al. might be a result of the gene editing happening before the first cell division occurred.

The edited embryos developed similarly to the control embryos, with $50 \%$ reaching an early stage of development known as the blastocyst, in which the embryos contain different cell types. This indicates that editing does not block development.

When injecting the editing components, the authors also included a nucleotide sequence containing wild-type MYBPC3 that could be used as a repair template by the editing machinery. The authors designed the repair template to encode the same amino acids as the wild-type maternal copy of the gene, but used some different nucleotides so that they could distinguish between repair using either the maternal copy of MYBPC3 or the introduced repair template.

Interestingly, when the authors analysed the $M Y B P C 3$ gene correction in the embryos, they found that only one of the 42 embryos that had wild-type MYBPC3 had used the introduced template for repair. Their results indicate that the wild-type maternal copy of $M Y B P C 3$ probably provided the repair template, rather than the introduced copy. This contrasts with the authors' observations in stem cells, in which the introduced nucleotide template was used for the repair process. The authors propose that the DNA-repair mechanism operating in an early embryo differs from that occurring in stem cells. In the embryo in which correction using the repair template could be detected, the template sequence was found in only a subset of cells, and the remaining cells in this embryo had probably been corrected using the maternal version of the gene.

Another hurdle for the use of CRISPR-Cas9 technology is the possibility of off-target edits that might arise if editing components bind to genomic regions that have high similarity to the sequence targeted by the RNA guide. Ma et al. did not detect a type of genetic alteration associated with off-target edits in the wild-type copy of $M Y B P C 3$, suggesting high-fidelity editing.

Potential off-target changes in other genes were evaluated. Strikingly, the off-target testing did not detect any mutations in the sequencing results from the embryonic cells, leading the authors to conclude that the targeting was accurate. This is an important finding because off-target edits have been described as a challenge for the use of CRISPR technology in the clinic. However, because the targeted gene sequences would vary, and variations 
would also be present in the sequences of each genome being edited, the risk of off-target events might vary for each individual case.

The authors investigated a situation in which one copy of a gene needed to be targeted for editing, and found that genetic repair relied on the other wild-type copy of the gene. However, in some diseases, both maternal and paternal copies of a gene are mutant. In this situation, the wild-type copy is absent, so a repair strategy would have to rely on the use of an introduced template sequence. Inducing double-stranded breaks in both copies of the mutant genes might enable such a template to be used; however, it is conceivable that this might lead to increased usage of the deleterious NHEJ pathway. In such a situation, it is probable that specific strategies to prevent NHEJ, such as the use of blocking agents, would be crucial. That strategy would also be beneficial even when only one copy of a gene needs to be targeted, because 16 out of 58 of the embryos analysed by Ma and colleagues showed signs of NHEJ activity. However, there is a clear need to ensure that such strategies do not result in other damaging effects on a developing embryo and its genome.

Although Ma and colleagues' demonstration that embryonic genome integrity is maintained after CRISPR-Cas9 editing is promising, further studies and optimization of this technology will be needed. These will have to confirm that the approach is safe in terms of criteria such as mosaicism, off-target editing and the detection of abnormalities in edited embryos before it can be used as a therapy for inherited diseases. Nevertheless, this study is paving the way as part of investigations that might lead to CRISPR-Cas9 reaching the clinic in the future. Until then, embryo genetic testing during IVF remains the standard way to prevent the transmission of inherited diseases in human embryos. .

\section{Nerges Winblad and Fredrik Lanner} are in the Department of Clinical Science, Intervention and Technology, Karolinska Institutet, 17177 Stockholm, Sweden, and at Karolinska Universitetssjukhuset, Stockholm. e-mail:fredrik.lanner@ki.se

1. https://www.clinicaltrials.gov/ct2/show/NCT0279 3856?term $=$ NCT02793856\&rank $=1$

2. National Academies of Sciences, Engineering, and Medicine. Human Genome Editing: Science, Ethics, and Governance (National Academies Press, 2017).

3. $\mathrm{Ma}, \mathrm{H}$. et al. Nature $\mathbf{5 4 8 ,}$ 413-419 (2017).

4. Carrier, L., Mearini, G., Stathopoulou, K. \& Cuello, F. Gene 573, 188-197 (2015).

5. Maron, B. J. J. Am. Med. Assoc. 287, 1308-1320 (2002).

6. Cong, L. et al. Science 339, 819-823 (2013).

7. Jinek, M. et al. Science 337, 816-821 (2012).

8. Liang, P. et al. Protein Cell 6, 363-372 (2015).

9. Kang, X. et al. J. Assist. Reprod. Genet. 33, 581-588 (2016).

10.Tang, L. et al. Mol. Genet. Genomics 292, 525-533 (2017).

11.Lin, S. et al. eLife 3, e04766 (2014).

This article was published online on 2 August 2017.

\section{Magnetic molecules back in the race}

\section{Single-molecule magnets have potential data-storage applications, but will need to work at a much higher temperature than has been possible. Two studies suggest that this goal could be met in the near future. SEE LETTER P.439}

\section{ROBERTA SESSOLI}

$\mathrm{M}$ ore than two decades ago, it was discovered that a single molecule could have two stable magnetic states - a property called magnetic bistability - at temperatures of a few kelvin ${ }^{1}$. In principle, such molecules could be used to store information, but this would require operating temperatures approaching at least that of liquid nitrogen (about $77 \mathrm{~K}$ ). On page 439 and writing in Angewandte Chemie, respectively, Goodwin et al. ${ }^{2}$ and Guo et al. ${ }^{3}$ report a remarkable advance in this direction. The authors demonstrate magnetic bistability in a molecule at temperatures as high as $60 \mathrm{~K}$, which is twice that achieved in previous studies ${ }^{4}$. consisting of a metal ion and organic ligands that exhibit magnetic bistability below a certain 'blocking' temperature. This temperature is mainly determined by the energy required to switch between two opposite orientations of the molecule's magnetic moment ${ }^{5}$. A key factor is the metal ion's magnetic anisotropy - the extent to which the ion's response to a magnetic field depends on the direction of the field.
Single-molecule magnets are compounds
Ions of lanthanide elements can have relatively large magnetic anisotropies and are therefore well suited to achieving high-temperature magnetic bistability. However, singlemolecule magnets that contain such ions need to be carefully designed to optimize the spatial distribution of the ligands' electrons with respect to that of the ion's electrons.

In the case of lanthanides whose electron distributions are oblate (squashed along the axial direction), such as terbium, dysprosium and holmium, an axial position of the ligands' electrons is particularly favourable for generating a sizeable magnetic anisotropy. However, lanthanide ions are often surrounded by many coordinated (neighbouring) ligand atoms in general, 8 to 10 - that are approximately spherically distributed around the ion.

A strictly axial coordination environment can be realized, for instance, by depositing a holmium atom on top of an oxygen atom in a thin layer of magnesium oxide ${ }^{6}$. In such a system, magnetic bistability has been observed for temperatures as high as $30 \mathrm{~K}$, allowing the holmium atom's magnetic moment to be detected and manipulated using scanningprobe-microscopy techniques ${ }^{7}$. Although

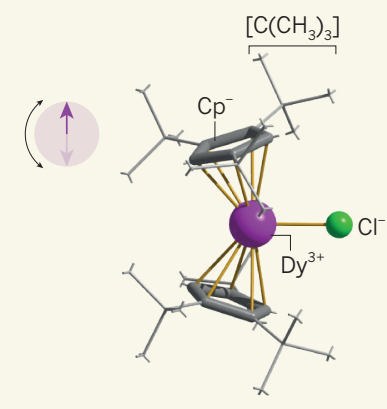

$\left[(\mathrm{Cp})_{2} \mathrm{DyCl}\right]$

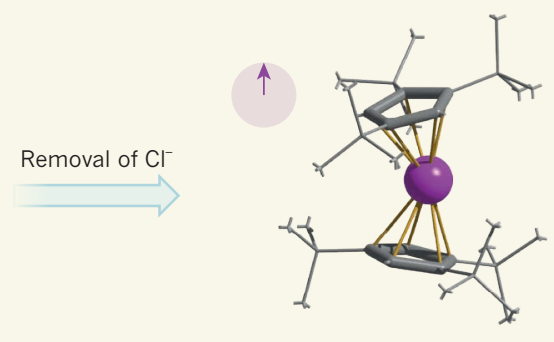

$\left[(C p)_{2} D\right]^{+}$
Figure 1 | Synthesis and structure of a single-molecule magnet. Goodwin et al. ${ }^{2}$ and Guo et al. ${ }^{3}$ report a molecule that has two stable magnetic states - a property called magnetic bistability - at temperatures approaching that required for data-storage applications. To synthesize their molecule, the authors sandwiched a dysprosium ion $\left(\mathrm{Dy}^{3+}\right)$ between two five-membered carbon rings called cyclopentadienyl anions $\left(\mathrm{Cp}^{-}\right)$. Each ring had three $t$-butyl $\left[\mathrm{C}\left(\mathrm{CH}_{3}\right)_{3}\right]$ substituents attached, and the authors added a chloride ion $\left(\mathrm{Cl}^{-}\right)$to the $\mathrm{Dy}^{3+}$ ion to create a neutral $\left[(\mathrm{Cp})_{2} \mathrm{DyCl}\right]$ complex. At this stage, the molecule's magnetic moment (purple arrow) was free to fluctuate between two opposite orientations. The authors then chemically removed the $\mathrm{Cl}^{-}$ion to produce a charged $\left[(\mathrm{Cp})_{2} \mathrm{Dy}\right]^{+}$complex. They showed that the molecule's magnetic moment was fixed in one of the two possible orientations, depending on the direction of a previously applied magnetic field (not shown). Grey and brown lines represent carboncarbon and metal-ligand bonds, respectively. 